\title{
Modelling of current and temperature effects on supercapacitors ageing. Part I: Review of driving phenomenology
}

\author{
Dimitri Torregrossa*, Mario Paolone \\ Distributed Electrical System Laboratory, Swiss Federal Institute of Technology, Lausanne, Switzerland
}

\section{A R T I C L E I N F O}

\section{Article history:}

Received 2 April 2015

Received in revised form 28 October 2015

Accepted 8 November 2015

Available online $\mathrm{xxx}$

\section{Keywords:}

Supercapacitor

Ageing

Life endurance test

Power cycling test

\begin{abstract}
A B S T R A C T
The paper proposes a study of the phenomenology driving the ageing mechanisms of electrochemical double layer supercapacitors (SCs) during their most common operating conditions. In particular, the paper focuses on the so-called life endurance (LE) and power cycling (PC) stresses. The paper first provides a description of the variables governing the SC behaviour at the microscopic scale (i.e., porosity of the electrode surface, diffusion and conductivity of the electrolyte). Then, the paper discusses the link between these variables and the ones describing the SC behaviour at the macroscopic scale (i.e., temperature of the electrolyte and delivered current). This analysis is applied to both LE and PC stresses and as well as to their combination. The discussions make reference to experimental results obtained at the Author's laboratory by means of a dedicated test bench.
\end{abstract}

(c) 2015 Published by Elsevier Ltd.

\section{Introduction}

Electric double layer capacitors, also known as supercapacitors (SCs), are electrochemical energy storage devices characterized by relatively low values of energy density (in the order of few $\mathrm{Wh} / \mathrm{kg}$ ), high values of power density (above $10 \mathrm{~kW} / \mathrm{kg}$ ) and extremely high cycling capabilities (i.e., above half million cycles). These characteristics are typical for devices operating in nominal operating conditions, namely below the device rated current and within the rated operating temperatures [1].

As known, a SC is essentially composed by two electrodes confining an electrolyte generally composed by an organic liquid (typical electrolyte are tetraethyl ammonium tetrafluoroborate TEABF4 and triethyl methylammonium tetrafluoroborate TEMABF4). The SC uses the electric field of the electric double layer established at the electrode-electrolyte interface to store energy. The current delivered from the SC is extracted from this double layer at the electrode-electrolyte interface. The so-called interactions losses between the electrode and the electrolyte are the fundamental ageing mechanism occurring in the device $[2,3]$.

From a macroscopic point of view, the SC is a device delivering energy to a load or receiving energy from a power generator. The external variables describing and influencing this energy transfer are: the voltage $v_{\mathrm{SC}}(t)$ across the SC, the external temperature $\tau_{\mathrm{SC}}(t)$ of the SC, the current $i_{S C}(t)$ delivered by the SC (or used to charge it).

\footnotetext{
* Corresponding author. Fax: +41 216934662.

E-mail address: dimitri.torregrossa@gmail.com (D. Torregrossa).
}

Macroscopically, the ageing process of a SC involves performancesfading associated to a decreased capability of storing (and delivering) a pre-defined amount of charge. In order to indirectly quantify the SC ageing process, a large part of the literature focused on the assessment of the so-called equivalent series resistance (ESR) and capacitance $\left(C_{\mathrm{SC}}\right)[1-15]$. The types of stresses proposed in these papers can be grouped in two main categories, namely: (a) life endurance (LE) (also called life calendar tests) [1-11] and (b) power-cycling (PC) stresses [10-15].

During a LE test, the $v_{\mathrm{SC}}(t)$ and the $T_{\mathrm{SC}}(t)$ are controlled in a specific environment in order to analyse their effects on the performances fading of the device. During these tests the SC is not delivering any current.

During a PC tests the environment temperature is kept constant to the rated value and the SC is charged/discharged with ani $i_{\mathrm{SC}}(t)$ of a given waveshape.

The results presented in the above works investigate the SC performances-fading with respect to $i_{\mathrm{SC}}(t)$ amplitudes comparable to the SC rated current and, to the best of the Authors knowledge, there are no contributions discussing the SC ageing with $i_{\mathrm{SC}}(t)$ amplitudes well beyond the SC rated values. Additionally, also the influence of high values of the environment temperature during combined PC and LE tests has not been addressed.

As known, from a microscopic point of view, a SC is an electrochemical device that stores charge using the double layer phenomenon [1]. At the interface between the electrode and the electrolyte, electrostatic interactions form a charge-layer around the electrode and, also, in the electrolyte. The current extraction from the electrode is based on the micro interaction between the 
porous electrode and the surrounding electrolyte. From this microscopic point of view, the ageing process is mainly related to the modification of the surface contact between the electrode and the electrolyte. The internal variables describing this surface interaction, and the consequent charge transfer, are: the porosity of the porous electrode $\Gamma$, the electrolyte conductivity $k$ and the viscosity $\lambda$ of the electrolyte, the charge diffusion coefficient $D$ and the size of the micropore structure on the electrode surface. Then, at microscopic level, the ageing of the SC is basically related to the lower interaction between electrode and electrolyte due to changes in $\Gamma, k$ and $\lambda[2,3]$.

In this respect, there are several works describing the micropore structure of the electrodes and its influence on the charge extraction (e.g., [2]) the temperature and voltage dependency with the conductivity and viscosity of the electrolyte (e.g., [14-16]). Although the analyses presented in these works are quite accurate, they are not framed in the context of SC applications. Indeed, they are not coupled with the high-power cycling that typically characterise the use of SCs. Consequently, the associated ageing mechanisms might be missed.

From this stand-point, the work presented in this paper aims at providing: (i) the phenomenological description of the ageing processes related to the most common categories of SC ageing stresses (i.e., LE and PC); (ii) the link between the local SC electrodes-electrolyte variables and the associated macroscopic ones during the ageing processes; (iii) the physical explanations supporting the time evolution of the SC external variables (ESR and $C_{\mathrm{SC}}$ ) during combined PC and LE stresses.

Once the physical explanations of the ageing process are described and validated, we propose in part II to quantify the evolution of the performances-fading by a suitable model for the evaluation of the device State-of-Health in terms of decrease of $C_{\mathrm{SC}}$.

The part I of the paper is structured as follows. Section 2 presents a short description of the physical processes driving the ageing mechanism of a SC; the links between local SC electrodeselectrolyte variables and external ones are proposed. Section 3 describes the ageing mechanisms driving the performances-fading during the targeted ageing tests; the quantification of the $C_{\mathrm{SC}}$ and ESR changes are supported by physical explanations. Conclusions summarize the contribution of the proposed work and introduce part II.

\section{Base phenomenology of the SC ageing process}

\subsection{Summary about the SC charge storage mechanism}

Before describing the SC ageing process, we here introduce the fundamental mechanisms that are used in these devices to store charges. This paragraph is intentionally introduced since we will use these concepts afterwards.

The modelling of the energy storage process associated to the double layer charge has been described in detail in [1]. As illustrated in [2,3], a SC is essentially composed by two carbon porous electrodes confining an electrolyte. The process of charge-transfer in a SC is composed by two sub-processes (see Fig. 1):

(a) the first one implies the ions diffusion within the electrolyte (electrolyte current);

(b) the second one refers to the electron diffusion within the electrodes toward the electric load connected to the SC itself (superficial electrode current).

Based on analysis given in Ref. [2], the superficial electrode current and the electrolyte one are given by the following

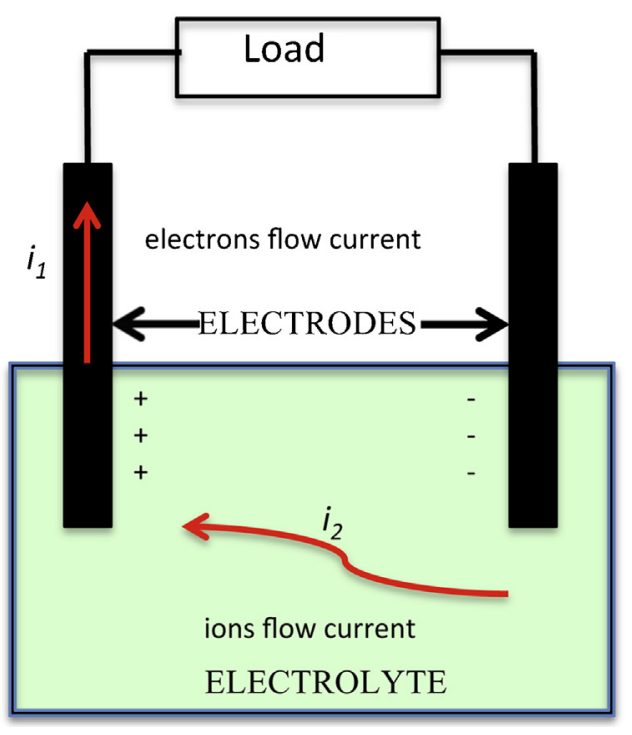

Fig. 1. Schematic representation of the charge transfer within a SC.

equations derived from the local-form of the Ohm's law:

$i_{1}=-\sigma \frac{\partial \phi_{1}}{\partial x}$

$i_{2}=-k \frac{\partial \phi_{2}}{\partial x}$

where $\sigma$ is the electrode conductivity, $k$ is the electrolyte conductivity, $\phi_{1}$ is the electrode potential, $\phi_{2}$ is the solution potential, $x$ is the coordinate associate with the width of the electrode or with the volume containing the electrolyte solution.

Eqs. (1) and (2) are derived from these hypotheses:

(a) the electrolyte concentration is uniform throughout the electrolyte itself. ${ }^{1}$ The solvent evaporation takes place with a long time constant.

(b) Ohmic losses are the main source associated to the heating of the targeted device and the environment temperature is not close to the electrolyte boiling point (typically ranging from $351.5 \mathrm{~K}$ to $358.5 \mathrm{~K}) .^{2}$

(c) the temperature is constant through the cell but it changes in time. $^{3}$

(d) The capacitance of the SC, along with the physical properties of the electrolyte, are constant over the range of possible SC voltage. It is also assumed that the potential difference $\phi_{1}-\phi_{2}$ is below the decomposition voltage of the electrolyte. As it will be explained later, the degradation phenomena associated to SC voltage values below the nominal one has a long time constant.

\subsection{SC ageing processes description at microscopic level}

By observing Eqs. (1) and (2), it is possible to state that, from a microscopic point of view, the ageing phenomena associated to the

This hypothesis is acceptable since, within the electrolyte of a SC, there are no redox reduction causing corrosion of the materials.

Such hypothesis is necessary in order to avoid the presence of other degradation phenomena.

The local over temperatures that can appear on the electrode electrolyte volume interface can be neglected. 
charge transfer of the SC depends on the electrode and electrolyte characteristics $\sigma$ and $k$. Additionally, these coefficients also affect the ion diffusion governed by the known Nernst-Plank equation [3].

As discussed in Ref. [3], the main SC degradation mechanism is the one associated to the process affecting the micro modifications of the pore structure of the electrode surfaces. This process involves four sub-degradations mechanisms taking place even in normal operating conditions (even if temperature, voltage and current are kept within the nominal ranges):

(a) a uniform current distribution within the electrode involving the so-called higher curvature on the $v_{\mathrm{SC}}(t)$ dynamic profile during the charge redistribution phase. That phenomenon indirectly causes a reduction of the current delivered by the SC.

(b) a decrease of the porosity of the electrode surface. The porosity of a carbon porous electrode is, in general, defined as follows:

$\Gamma=\frac{\lambda}{\lambda+\delta}$

where $\lambda$ is the average ratio of the pore on the electrode surface, $\delta$ is the average thickness distance between two neighbouring pores (see Fig. 2a). In Fig. 2a the pore structure is schematically represented by an average radius pore distribution with an average thickness distance. Actually, the electrode surface consists of large, irregular particles of carbon involving a specific pore distribution characterized by an internal surface area $\left(S_{\text {INT }}\right)$ and an external one $\left(S_{\text {EXT }}\right)$ (see Fig. 2b). The pore can be classified in three main categories of pores: macropore (mean radius higher than $50 \mathrm{~nm}$ ), mesopore (mean radius between 2 and $50 \mathrm{~nm}$ ) and micropore (mean radius lower than $2 \mathrm{~nm}$ ) (i.e., Ref. [2]).

The electrodes are preventively treated in order to reach an optimal macropore distribution and optimal size aiming at allocating the ions charge coming from the electrolyte. In this respect, there is an optimal value of porosity that is chosen by the SC manufacturer. The porosity of the electrode depends indirectly on the pore distribution generally expressed as:

$\Gamma \sim \frac{S_{\mathrm{EXT}}}{S_{\mathrm{INT}}}$

It should be underlined that only the micropores contribute to $S_{\text {INT }}$ as well as meso and macropore contribute to $S_{\text {EXT. }}{ }^{4}$

(c) Decrease of the electrolyte conductivity. This quantity, around the electrode surface, depends on the electrode porosity with the following approximated equations (e.g., Ref. [2]):

$k=k_{0} \sqrt[3]{\Gamma^{2}}$

where $k_{0}$ is the bulk conductivity of the electrolyte. It is clear that a decrease of porosity lead to an important decrease of the electrolyte conductivity.

a)

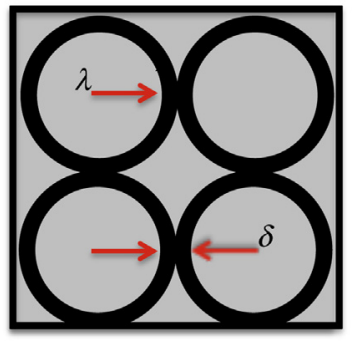

b)

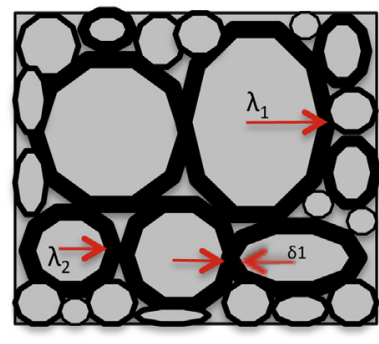

Fig. 2. (a) Schematic average representation of the electrode surface structure; (b) Schematic representation of the electrode surface structure. (d) Decrease of the ion diffusion coefficient. This quantity, around the electrolyte, depends on the electrode porosity with the following approximated equation (e.g., Ref. [2]):

$D=D_{0} \sqrt[3]{\Gamma^{2}}$

where $D_{0}$ is the bulk ion diffusion coefficient of the electrolyte. It is clear that a decrease of porosity leads to an important decrease of the diffusion coefficient.

It is worth nothing that the above parameters $\Gamma, k, D$ are temperature-dependent. Additionally, the Stokes-Einstein equation gives the relation between the diffusion coefficient and the temperature $T$ in a classical electrolyte solution:

$D=\frac{k_{\mathrm{B}} T}{6 \pi r \lambda}$

where $k_{\mathrm{B}}$ is the Boltzamn constant and $r$ is the mean radius of the spherical particle. The study proposed in this work neglects the electrode conductivity temperature changes since they appear for temperatures above $500{ }^{\circ} \mathrm{C}^{5}$

\subsection{SC ageing processes description at macroscopic level}

From a macroscopic point of view, the SC is a device able to deliver energy $E_{\mathrm{SC}}$ and power $P_{\mathrm{SC}}$ (max value of $P_{\mathrm{SC}}$ for a given $V_{\mathrm{SC}}$ ) that can be uniquely linked to external observable state quantities: $v_{\mathrm{SC}}(t), i_{\mathrm{SC}}(t)$ and $\tau_{\mathrm{SC}}(t)[17]$ :

$E_{\mathrm{SC}}=\frac{1}{2} C_{\mathrm{SC}} V_{\mathrm{SC}}^{2}$

$P_{\mathrm{SC}}=\frac{V_{\mathrm{SC}}^{2}}{4 \mathrm{ESR}}$

By observing Eqs. (7) and (8), from a macroscopic point of view, the ageing phenomena associated to the energy and power transfers of the SC is associated to the capacitance fading and ESR increase.

The capacitance $C_{\mathrm{SC}}$ is related to the capability of the targeted device to both store and deliver charges. In this respect, in Ref. [2] it has been introduced the following parameter, called RAC (relative available charge):

$\mathrm{RAC}=\frac{Q_{\mathrm{EXT}}}{Q_{\mathrm{MAX}}}=\frac{\int_{T_{D}} i_{\mathrm{SC}}(t) \mathrm{d} t}{Q_{\mathrm{MAX}}}$

where $Q_{\text {EXT }}$ is the amount of charge extracted from the SC during the time $T_{D}$ (being $T_{D}$ the discharging time needed to reach a null voltage across the SC terminals) and with the current profile $i_{\mathrm{SC}}(t)$, $Q_{\text {MAX }}$ is the maximum amount of charge that theoretically can be stored inside the SC.

If $Q_{\text {MAX }}$ is the amount of charge stored previously in the SC in order to reach the required value of voltage (for example $V_{\mathrm{N}}$ ), the definition of RAC is equal to that one of $C_{\mathrm{SC}}$ [20]. The internal degradations of the materials composing the $S C$ involve a decrease of both $Q_{\mathrm{EXT}}$ and $Q_{\mathrm{MAX}}$. These degradations depend indirectly on the values of several external variables, such as: the delivered

\footnotetext{
${ }^{4}$ Eq. (4) is based on an extension of the general model of porous electrode developed by Newman and Tiedemann. The pore size distribution is indirectly taken into account via the definition of the porosity in equation. We neglected the effect of the ageing on pore size distribution.

We neglect the effect on the separator since the experimental result illustrated in Ref. [20] show that the separator is affected only if the SC voltage or the SC
} temperature are above the nominal values. 
current $i_{\mathrm{SC}}(t)$, the voltage $v_{\mathrm{SC}}(t)$ and the average SC temperature $\tau_{\mathrm{SC}}(t)$.

Based on the considerations made in the previous sub-section, the RAC value directly depends on the value of superficial electrode current and the electrolyte current $\left(i_{1}, i_{2}\right)$. Consequently, RAC depends on the conductivity of the electrode, $\sigma$, and the electrolyte, $k$, and on the electrode-electrolyte interface interactions. In this respect, RAC depends indirectly on the diffusion charge coefficient $(D)$ of the electrolyte and on the porosity $(\Gamma)$ of the electrodes surface.

Therefore, we can write the generic link:

$$
\begin{aligned}
C_{\mathrm{SC}=} f_{1}(\mathrm{RAC}) & \approx f_{2}\left(i_{1}, i_{2}\right) \approx f_{3}(\sigma, k) \approx f_{4}(\sigma, \Gamma, D) \\
& \approx f_{5}(\sigma, \Gamma, D, T)
\end{aligned}
$$

Concerning the ESR, it is formally defined as the ratio between the offset on $v_{\mathrm{SC}}(t)$ appearing after stopping a charge or discharge phase and the associated current $i_{\mathrm{SC}}(t)$. Based on the consideration reported in [1], the ESR is composed by the following main contributions:

- the resistance of the electrode itself $R_{\text {ELECTRODE }}$ (note that this value is not affected for temperature below $500^{\circ} \mathrm{C}[1] .^{6}$

- The resistance between the electrode-electrolyte interface $R_{\text {INTERFACE. This quantity depends strongly to the micropore }}$ modifications of the electrode surfaces due to the ageing process associated to the extraction of the current. The surface contact can be modelled as a large amount of micro contact paths though which the ions can flow and be absorbed by the electrode. Each of these paths has a resistance value given by the following equation:

$R_{i}=\frac{1 l_{\mathrm{C}}}{k S_{\mathrm{C}}}$

where $S_{\mathrm{C}}$ is the surface contact and $l_{\mathrm{C}}$ the length of the path.

- The resistance of the electrolyte $R_{\text {ELECTROlyte. This quantity }}$ depends strongly on the electrolyte conductivity and its viscosity. Based on the experimental investigations reported in [16-18] the dependency of the electrolyte conductivity $k$ as a function of its temperature can be described by the following equation:

$k=k_{0} \exp \left[-\frac{B}{T-T_{0}}\right]$

where $B$ is a constant depending on the composition of the electrolyte, $T$ is the temperature of the electrolyte, $T_{0}$ is the nominal temperature of the electrolyte for which the bulk conductivity is reported.

The conductivity of the electrolyte inversely depends on the viscosity of the electrolyte itself. As known, this latest quantity increase with the increase of the electrolyte temperature since the viscosity decreases and the ion mobility increases (the diffusion coefficient $D$ has the temperature dependency described by Eq. (7)).

Among the three of the above-listed resistances, $R_{\text {INTERFACE }}$ and $R_{\text {ELECTROLYTE }}$ are the most important elements contributing to the ESR since they depend directly on the micro pore modifications of the electrode surfaces along with the electrolyte properties. These modifications, as it will be detailed in the next section, are the main

\footnotetext{
${ }^{6}$ Note that the electrode resistivity is characterized by a temperature coefficient that is two orders of magnitudes lower than copper.
}

ones accounted to represent the ageing processes of this specific quantity.

Based on the above considerations, since the main contribution to the ESR comes from the resistance of the electrolyte, it is possible to state that, in a first approximation, the $P_{\mathrm{SC}}$ depends on $R_{\text {ELECTROLYTE }}$ only. With this last quantity that depends mainly on the conductivity of the electrolyte. Based on Eqs. (5), (6) and (13), the conductivity depends on the porosity of the electrode and on the SC temperature. Finally, we can approximate the dependency of $P_{\mathrm{SC}}$ only as a function of the micropore structure of the electrode surface:

$P_{\mathrm{SC}}=g_{1}(\mathrm{ESR}) \approx g_{2}(k) \approx g_{3}(\Gamma) \approx g_{4}(\lambda, \delta) \approx g_{5}(\lambda, \delta, T)$

The links generically described by Eqs. (11) and (14) are important since they shown that the most common external parameters used for evaluate the ageing of the SC are physically related to the degradation of the internal components (electrolyte and electrode surface).

The links shown in these two equations are the base for the physical interpretations of the performances losses during a PC, LE and combined PC and LE stress. In this respect, in the second part of this work the Eq. (11) will be inferred experimentally.

\section{Description of the ageing mechanisms during combined PC and LE tests}

\subsection{Experimental test bench}

The complete description of the experimental bench test along with the procedure to compute the $C_{\mathrm{SC}}$ and ESR is illustrated in Refs. [19,20]. In what follows, a brief description of the experimental test bench, adopted devices and testing conditions are given.

The electric double layer capacitors used in this investigation were all supplied by the same manufacturer. The obtained results have been experimentally inferred from a group of SCs of $365 \mathrm{~F}$ belonging to the same set. The devices have a capacity of $365 \mathrm{~F}$ and operating voltage of 0 up to $2.7 \mathrm{~V}$. It was established from the manufacturers that the capacitor electrolyte is composed of 20-30\% Tetraethylammonium Tetrafluoroborate (TEA BF4) in Acetonitrile. The electrodes comprise porous activated carbon with carbon black as a conductivity modifier possibly bound with a patented n-vinyl acetamide polymer binder containing butadiene styrene nanoparticles (note that the binder could not be confirmed due to IP restrictions). This carbon composite is distributed on both sides of an aluminium foil current collector and housed in aluminium casing. All connections are aluminium, and the separator is thin porous paper.

The power test setup is composed of four main elements (see Fig. 3): (i) a climatic chamber hosting the targeted SC with operating temperatures in the range $-40-360^{\circ} \mathrm{C}$. In steady state conditions, the temperature ripple is of $\pm 0.2^{\circ} \mathrm{C}$. (ii) a power source working in the following $V / I$ ranges $0-80 \mathrm{~V}, 0-120 \mathrm{~A}$; (iii) an

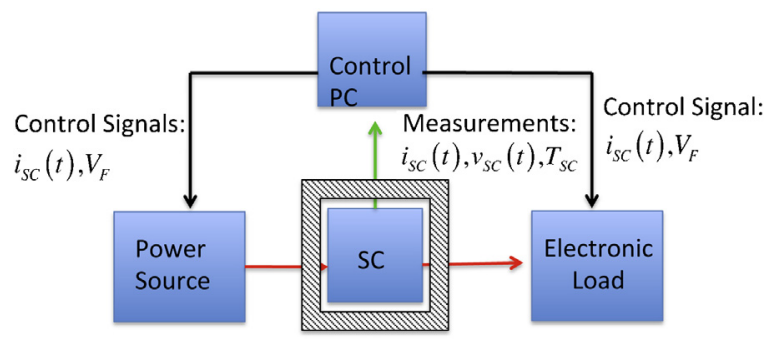

Fig. 3. Block scheme of the experimental test setup. 
electronic load working in the following $V / I$ ranges $0-80 \mathrm{~V}, 0-$ $200 \mathrm{~A}$; (iv) control PC where, a suitable realized software developed using the LabView programming environment, is able to perform the monitoring and control the whole system.

The electronic load and power supply were characterized by an overall bandwidth from DC to $1 \mathrm{kHz}$. The supercapacitor voltage $\left(V_{\mathrm{SC}}\right)$ was directly sampled using an analog-to-digital converter operating a 16 bits with a maximum sampling frequency of $100 \mathrm{kHz}$ and characterized by a bandwidth from DC to $100 \mathrm{kHz}$ $(-3 \mathrm{~dB})$ with an overall accuracy of $1.5 \mathrm{mV}$. The supercapacitor current $\left(I_{\mathrm{SC}}\right)$ was measured using a dedicated hall-effect sensor characterized by an overall bandwidth from DC to $100 \mathrm{kHz}(-3 \mathrm{~dB})$. Also for the $I_{\mathrm{SC}}$, the analog output of the current sensor was sampled with a analog-to-digital convert with the same characteristics as the one used for the $V_{\mathrm{SC}}$.

The procedure for assessing the SC parameters is structured as follows: (i) the $\mathrm{SC}$ is charged with the nominal current of $36 \mathrm{~A}$ until the $S C$ reaches a final voltage value $V_{\mathrm{F}}=2.7 \mathrm{~V}$, during this phase the SC input electrode resistance ESR, is evaluated by measuring the voltage drop across the SC terminals taking place at the beginning of the test when the step current is applied; (ii) the power generator is turned off and the charge redistribution phenomenon is observed and recorded for $20 \mathrm{~min}$, (iii) the SC is discharged with a constant resistance $R_{\text {load }}$ equal to $0.1 \Omega$ until the $V_{\mathrm{SC}}$ reaches $100 \mathrm{mV}$. During this last phase the capacitance is computed as follows:

$C_{\mathrm{SC}}=\frac{\Delta Q_{\mathrm{DISCH}}}{\Delta V_{\mathrm{DISCH}}}$

where $\Delta Q_{\mathrm{DISCH}}$ is the time integral of the current delivered by the SC and $\Delta V_{\text {DISCH }}$ the voltage variation during the phase (iii).

Each type of experimental investigations has been carried out for 4 SC samples belonging to the same production lot.

\subsection{Power cycling ageing stress}

As described in Ref. [10], the main factor influencing the SC ageing mechanisms during a PC test (characterised by a defined charge/discharge cycle) is the RMS value of the current. Indeed, the degradation of the SC during a PC test is related to the amount of charges flowing through the electrolyte-electrode interface involving a de-cohesion of the carbon composing some parts of the electrodes.

The phenomenological investigations of the above-described ageing mechanism have not been completely addressed by the literature and, consequently, there is not unanimous consensus on the reason at the base of the SC performances fading.

As it has been already briefly summarised in the introduction, the SC electrodes have a porous surface with different pore sizes allowing for the absorption of the ion flow of the electrolyte and the consequently absorption from the electrode itself. As illustrated in Ref. [2] the widest pores, i.e. macropores characterised by a width around $50 \mathrm{~nm}$, are the most important ones for the charge-storage. As a consequence, it is plausible to assume that an increase of porosity always involves a benefit in terms of capacitance increase. However, this assumption it is not true since there is a certain optimal value of porosity $\Gamma_{\text {OPT }}$ around which the $C_{\mathrm{SC}}$ starts to decrease. The reasons at the base of this statement are given in what follows. It is worth nothing that, based on Eq. (11), the $C_{\mathrm{SC}}$ is mainly function of the electrolyte conductivity that, in turn, depends on the porosity and on the $T_{\mathrm{Sc}}$. The external parameter appearing in Eq. (10) is the RAC that indirectly takes into account all the micropore modifications and all the electrolyte properties changes. The analysis reported in [2] qualitatively evaluates the impact of porosity on the RAC value. It is reported

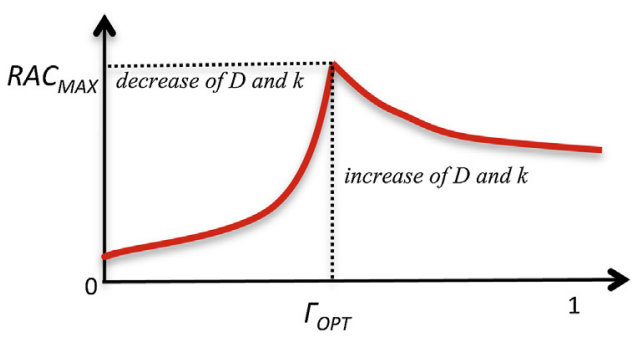

Fig. 4. Qualitative evolution of RAC vs the electrodes porosity.

that there is an optimal value of porosity for which the RAC has the maximum value and consequently the $C_{\mathrm{SC}}$. Fig. 4 illustrates qualitatively the evolution of RAC versus the porosity. This evolution graphically summarizes the considerations and analysis reported in Ref. [2]. As it can be deduced from Eq. (3), the porosity ranges from zero (the electrode is no porous at all) to one (the electrode is perfectly structured with pore of optimal size and the thickness between two neighbouring pores is quite negligible). As it can be observed, there is an optimal value of porosity $\Gamma_{\text {OPT }}$ for which the value of RAC is maximised. For porosity values lower than $\Gamma_{\text {OPT }}$ the average value $\lambda$ increases, meaning a higher $S_{\mathrm{EXT}}$, and it involves a consequent higher interaction between electrode surface and electrolyte ions. However, based on Eq. (6), the diffusion coefficient, along with the electrolyte conductivity, are both decreasing and they are leading to a lower amount of charge that can be extracted from the SC. These two conflicting effects are not balancing each other and the one related to the decrease of RAC is generally predominant. For this reason, the RAC decreases if $\Gamma<\Gamma_{\text {OPT }}$. On the other hands, if the porosity is higher than $\Gamma_{\text {OPT }}$, the average value $\lambda$ is decreasing, meaning a lower $S_{\mathrm{EXT}}$, consequently, it involves a lower interaction between electrode surface and electrolyte ions. ${ }^{7}$ This negative effect is partially compensated by the increase of $D$ and $\square$ due to the increase of porosity. These coupling effects produce a decrease of RAC of lower entity compared to that one estimated for $\Gamma<\Gamma_{\text {OPT }}$.

In what follows the paper discusses what happens in the SC during a PC stress. The capacitance losses and ESR increase depicted in the majority of the ageing-analysis papers can be explained based on the above considerations on porosity changes.

Actually, during a PC test, due to the extraction of the current, there are several impurities coming from the de-cohesion and micro-cracking of carbon electrode particles $[4,5,24-30] .^{8}$ This phenomenon is higher if the extracted current is several time higher than the nominal one (as in the work here presented). These impurities, along with those already distributed in the electrolyte, such as metal particles and water [19], are filling-elements of the pores of the electrode surfaces and they increase the width of the surface contact $\left(l_{C}\right.$ in Eq. (12)) and decrease the electrode surface itself $\left(S_{C}\right.$ in Eq. (12)) This involves a reduction of the average radius $\lambda \square \square \square$, consequently, produces an increase of porosity (we are moving on the right-hand side of Fig. 4). Additionally, during a PC ageing test, due to the internal energy losses associated to the energy transfer, there is heat production involving increase of temperature especially located in correspondence of the electrodeelectrolyte interface. Based on Eq. (13) an increase of temperature leads to an increase of electrolyte conductivity and diffusion coefficient of the electrolyte. These latest effects involve an

${ }^{7}$ The size of the pore is not that one allowing the optimal allocation and absorption of the electrolyte ions.

${ }^{8}$ Based on the available literature we can assume that this effect does not depend on the type of electrochemical energy storage system but on the type of the electrodes (graphite in this work) 


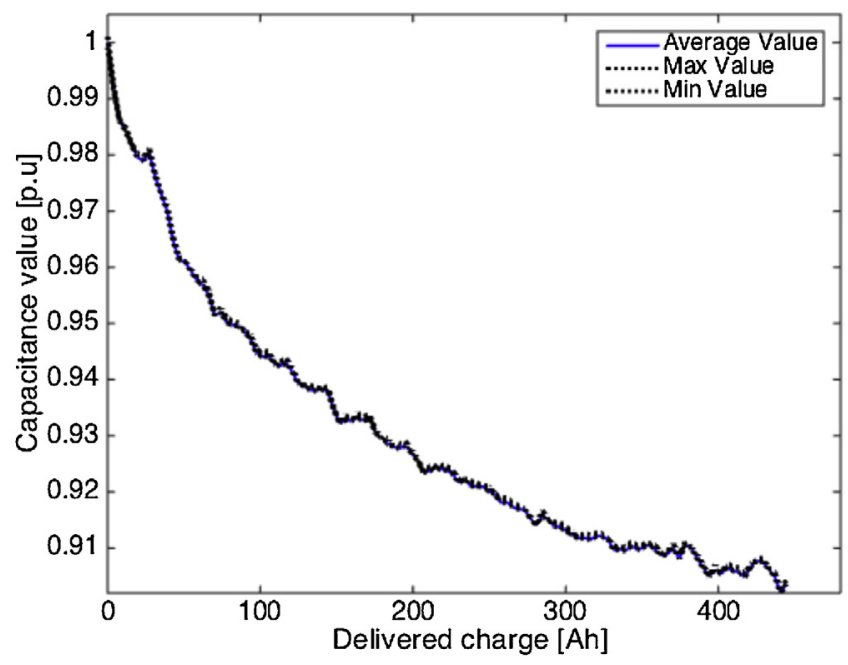

Fig. 5. $C_{\mathrm{SC}}$ evolution as a function of the delivered charge (in $\mathrm{Ah}$ ) during a PC test at 298.15 K in p.u. of starting value. Averaged values among 4 samples. Highest bound magnitude of $0.2 \%$. Average starting value of $C_{\mathrm{SC}}$ of $351 \mathrm{~F}$.

increase of the electrolyte and electrode current and, consequently, an increase of the extracted current. Globally, the $C_{\mathrm{SC}}$ is reducing its value. In this respect, Fig. 5 illustrates the evolution of the SC capacitance versus the charge delivered by the SC during a PC at 298.15 K. The detail related to the setup and the current profiles used for ageing the SC are provided in [19]. In Fig. 5 it is possible to observe a different rate of capacitance change during the PC ageing test. Indeed, at the beginning of the test, for the first $20 \mathrm{Ah}$ delivered by the SC the capacitance rate-of-change is more important than that one measured for the same amount of delivered charge at the end of the test. This different behaviour can be explained as follows. During the first cycles of a PC test, the porosity of the electrode surface, according with Fig. 4, it is varying quickly since the first impurities generated by the current extraction quickly fill part of the macro pores. Then, once the temperature of volume associated to the electrode-electrolyte interface is increased, the conductivity and the ion mobility increase as well and they are reducing the negative effect associated to the extraction current.

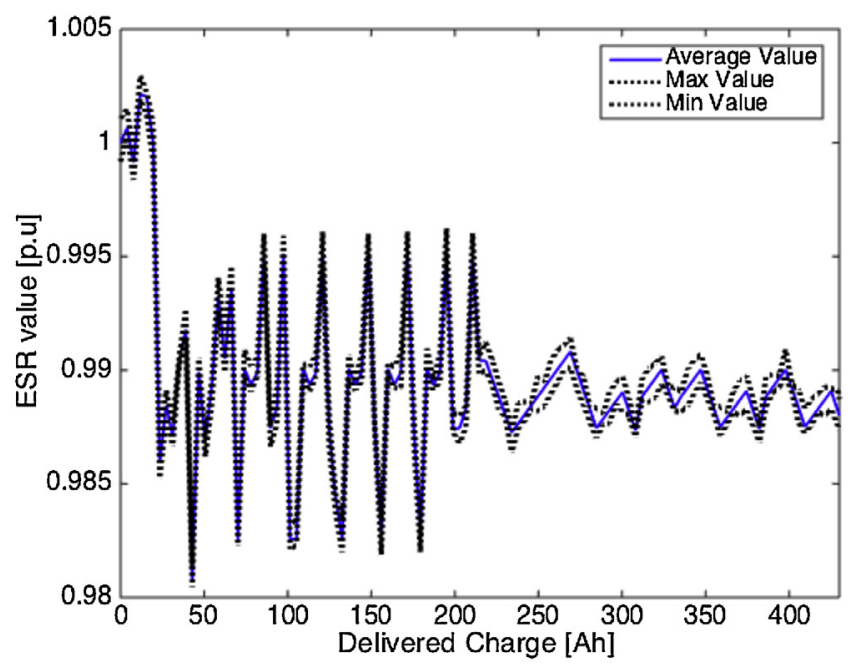

Fig. 6. ESR evolution as a function of the delivered charge (in Ah) during a PC test at 298.15 K in p.u. of starting value. Averaged values among 4 samples. Highest bound magnitude of $0.19 \%$. Average starting value of ESR of $3.1 \mathrm{~m} \Omega$.
Concerning the second external parameter influencing the power transfer of the SC, i.e. the ESR, it has been experimentally investigated that it does not change considerably during a PC test at nominal temperature [19]. (see Fig. 6). Actually, as already discussed in section II, ESR is mainly composed by $R_{\text {INTERFACE }}$ and

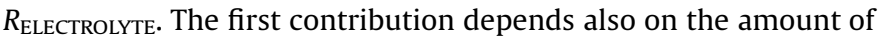
impurities filling the pores, increasing the width interstices between pores and decreasing locally the conductivity. These impurities compose a blocking element of the electrode-electrolyte surface contact (i.e., a decrease of $S_{\mathrm{C}}$ ) and lead to an increase of the length $l_{C}$ of the micro-contact paths for the ion electrolyte current. Consequently, the $R_{\text {INTERFACE }}$ increases. The extraction current allows for the remove of these impurities and reduction of the length $l_{\mathrm{C}}$. It is important to notice once more that the current extraction has a positive effect on the ESR but, on the other hands, it reduces the macropore size increasing the porosity of the electrode surface. The second contribution, i.e $R_{\text {ELECTROLYTE, is }}$ mainly related to the electrolyte conductivity changes. Since during the PC test there is an over temperature generally in the range of 4-6 K, the electrolyte conductivity increases its value and it possible to observe experimentally a slight decrease of the ESR (see Fig. 6).

The evolution of the ESR and $C_{\mathrm{SC}}$ at higher temperature are reported in sub-section 3.4 .

\subsection{Life endurance ageing test}

As described in Refs. [4-11] the main factors influencing the SC ageing mechanism during a LE ageing test are the temperature $T_{\mathrm{SC}}(t)$ and voltage $v_{\mathrm{SC}}(t)$. Indeed, it is possible to infer, based on the analysis reported in [1-23] that the degradation of the SC during a LE test is mainly related to the electrolyte properties changes (concentration, conductivity and viscosity) along with modifications of the porous electrodes structure. It is worth nothing that, even if the SC is not delivering any current but is stressed with voltage and temperature below the rated values, there is a certain degradation of the electrolyte. This degradation involves the generation of new impurities (as water) that limit the access to the pores [19]. On the other hands, in the analysis reported in Ref. [16] it is experimentally demonstrated that the viscosity of organic electrolyte decrease for temperature increase along with an higher ion mobility due to the associated increase of electrolyte conductivity. It is possible to assume that, if the SC temperature is lower than the boiling point of the electrolyte, the associate properties changes are not involving an important ageing of the SC electrolyte. Based on these considerations, in what follows we are describing which are the driving mechanisms leading to a capacitance decrease and a ESR increase during a LE stress.

As briefly introduced, during a LE stress both voltage and temperature are the ageing factors. Concerning the voltage stress, if it is kept below $3.75 \mathrm{~V}$, is not involving any direct ageing of the electrolyte since it involves an additional over temperature of the electrolyte. Since in this work the aim is to investigate the effect of over current and over temperature on the ageing mechanism, the $v_{\mathrm{SC}}(t)$ is kept under the nominal value. ${ }^{9}$

Even if the increase of the temperature can be appointed as a benefit factor increasing the electrolyte conductivity, it is important to remind that the electrolyte inside the SC is generally composed by an organic solution in which the solvent is the acetonitrile (AN) and the salt is the tetrafluoroborate (TEABF4) that is dissociated in anions $\left(\mathrm{BF}^{-}\right)$and cations $\left(\mathrm{TEA}^{+}\right)$. These ions are

\footnotetext{
${ }^{9}$ It is worth underlining that the presence of the voltage, even if under the nominal value, involves an additional over temperature into the electrolyte and consequently it involves an increase of the associated ageing process.
} 


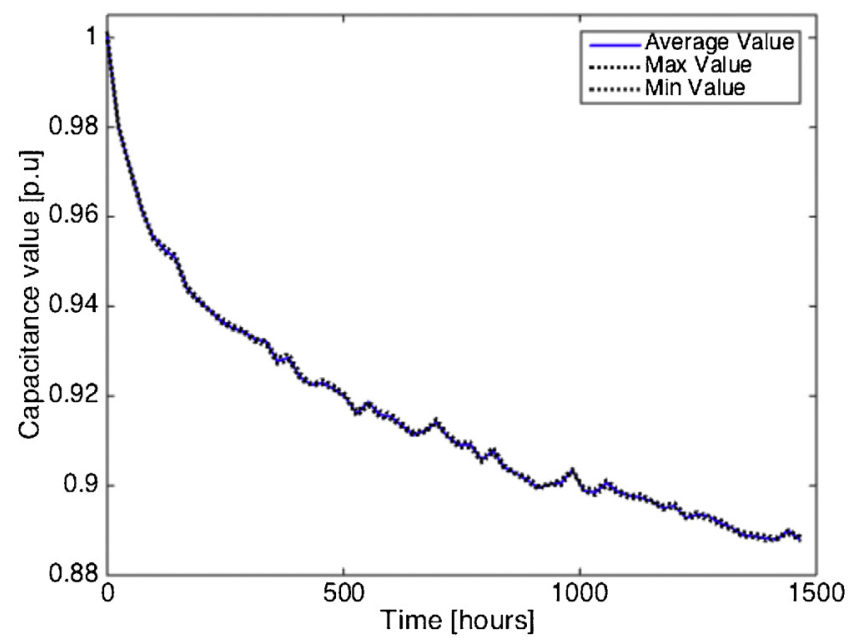

Fig. 7. $C_{\mathrm{SC}}$ evolution as a function of the time (in h) during a LE test at $328.15 \mathrm{~K}$ in p.u. of starting value. Averaged values among 4 samples. Highest bound magnitude of $0.23 \%$. Average starting value of $C_{\mathrm{SC}}$ of $349.5 \mathrm{~F}$.

essential for the electrolyte current and they are the ions absorbed by the electrode and involving the electrode current. The AN, even at $293 \mathrm{~K}$, starts to evaporate. From this standpoint, the increase of the electrolyte temperature leads to a loss of solvent that is evaporating and going out from the SC (indeed, SCs are, in general, not perfectly sealed). In such conditions the stoichiometry ratio between solvent and salt is not anymore granted and there is a certain quantity of salt that is not dissolved and creating impurities whose effects are described below. Consequently, there is a minor amount of ions available for the charge transfer. ${ }^{10}$

A LE test has been performed at $328.15 \mathrm{~K}$ during 60 days. The experimental evaluation of the $C_{\mathrm{SC}}$ and ESR has been performed by following the procedure described in Ref. [19]. It is important to underline that the above parameters have been evaluated each $24 \mathrm{~h}$. During this specific phase the SC is charged and discharged during two minutes and consequently the integrity of a pure LE is violated. In fact, the charge delivered by the SC during the performance test modifies the quantity of impurities deposited on the electrodes and involves a temporary increase of the electrolyte temperature. Due to the low increment of temperature (below $1 \mathrm{~K}$ ) along with the low duration of the charging/discharging phase, it is reasonable to assume that the ageing process is not affected by the evaluation parameters phase. After this latest phase, the $v_{\mathrm{SC}}(t)$ is evolving as a function of the associated redistribution phenomenon [19].

Fig. 7 illustrates the evolution of the $C_{\mathrm{SC}}$ during this LE test. As anticipated, the $C_{\mathrm{SC}}$ is reducing its value.

Concerning the second macroscopic parameter, i.e. the ESR, in Refs. [4-10] it has experimentally assessed its increase during a LE test. This result is confirmed also in our test as shown by Fig. 8. It is reported the time evolution of the ESR during the same LE test at $298.15 \mathrm{~K}$. With respect to this ESR behaviour, it is worth observing that the contribution $R_{\text {ELECTROLYTE }}$ decreases its value since, with higher temperatures, the conductivity of the electrolyte increases as well as its viscosity. The contribution $R_{\text {INTERFACE }}$ increase its value since the increase of the temperature, as already stated, increases the quantity of impurities filling the micropore of the electrode surface. In this respect, even if the local conductivity of the electrode electrolyte interface decreases the $R_{\text {INTERFACE }}$ surface increases (see

\footnotetext{
${ }^{10}$ In the appendix of the second part of this work, an experimental quantification of the solvent evaporation during a combined LE and PC stress is described.
}

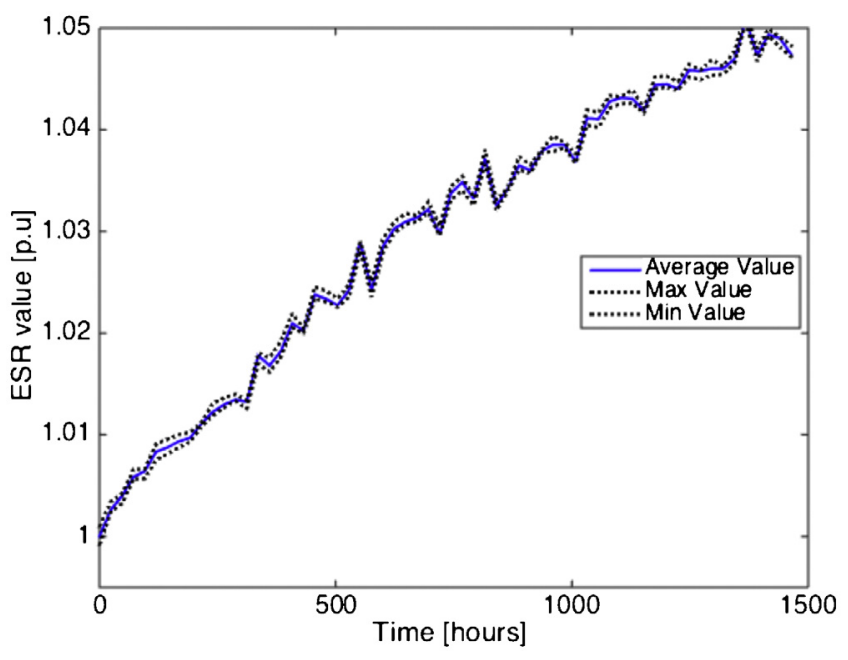

Fig. 8. ESR evolution as a function of the time (in h) during a LE test at $328.15 \mathrm{~K}$ in p. u. of starting value. Averaged values among 4 samples. Highest bound magnitude of $0.24 \%$. Average starting value of ESR of $3 \mathrm{~m} \Omega$.

Eq. (11)). Globally, the effect of the temperature during a LE test, along with the absence of current extraction, is to largely increase the ESR.

\subsection{Combined high temperature LE and high pulsed current PC stress}

SC applications generally require operating conditions involving charge/discharge currents and/or nominal temperature beyond the rated values. In this respect, it can be useful to investigate the ageing mechanism during combined LE and PC stress. Several works have already experimentally investigated the performances-fading of the SC during combined LE and PC test [12-15]. However, in these works the current delivered by the SC is in the same order of magnitude of the nominal current.

Especially during this specific type of stress, the performancesfading are not originating from a single cause but rather from a complex interaction of different ageing mechanisms. Indeed, during PC stress the main ageing process associated to the current extraction is the porosity changes on the electrode surfaces; for the LE stress the main ageing process is the one associate to the loss of solvent and the impurities filling the macropores of the electrode

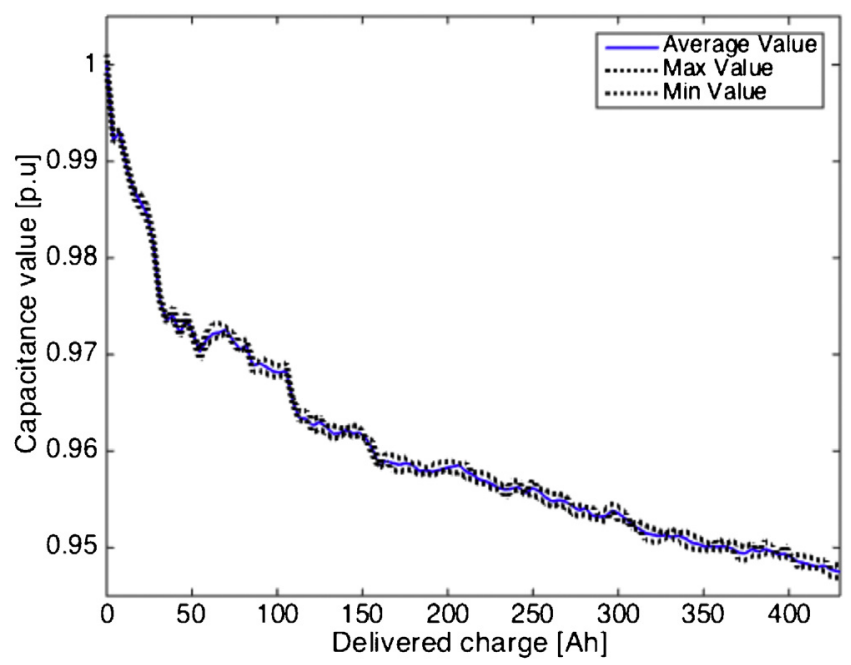

Fig. 9. $C_{\mathrm{SC}}$ evolution as a function of the delivered charge (in Ah) during a combined LE and PC test at $328.15 \mathrm{~K}$ in p.u. of starting value. Averaged values among 4 samples. Highest bound magnitude of $0.22 \%$. Average starting value of $C_{\mathrm{SC}}$ of $347.3 \mathrm{~F}$. 


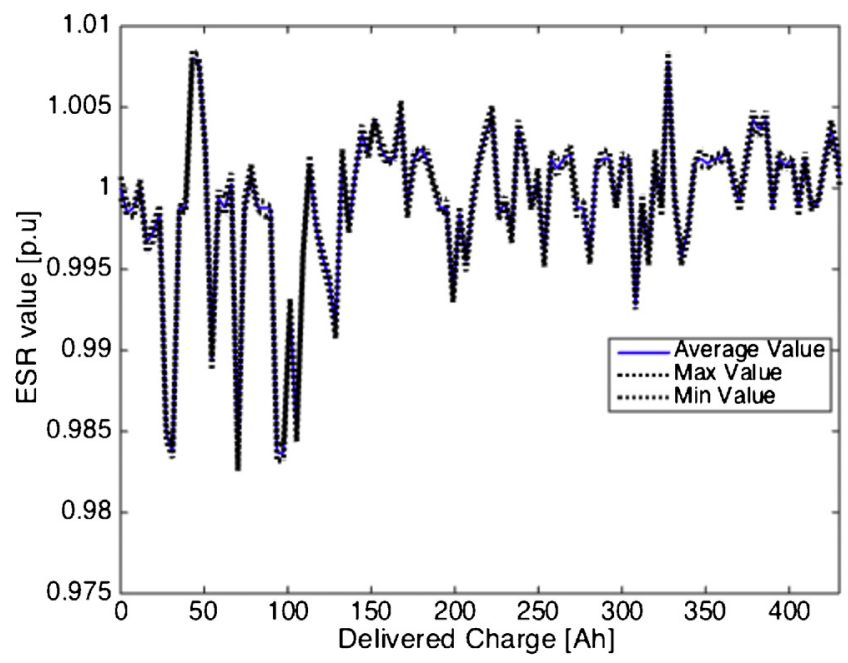

Fig. 10. ESR evolution as a function of the delivered charge (in Ah) during a combined LE and PC test at $328.15 \mathrm{~K}$ in p.u of starting value. Averaged values among 4 samples. Highest bound magnitude of $0.21 \%$. Average starting value of ESR of $2.91 \mathrm{~m} \Omega$.

surface. During a combined PC and LE stress, the above ageing mechanisms are acting together and the results, as it can be expected, is to increase the performances-fading quantified during a PC and a LE test. Before illustrating and discussing the performances fading during combined high temperature LE and PC stresses, Figs. 9 and 10 show the evolution of the $C_{\mathrm{SC}}$ and the ESR during at combined LE and PC stress at $308.15 \mathrm{~K}$, respectively. By observing these figures and comparing them with the results shows in Figs. 5 and 6 (at $298.15 \mathrm{~K}$ ) it is it possible to make the following comments:

- the $C_{\mathrm{SC}}$ fading is evidently less important at $308.15 \mathrm{~K}$. This is due to the fact that, qualitatively, at higher temperatures the electrolyte conductivity increase and the viscosity decrease;

- The ESR is almost constant at $308.15 \mathrm{~K}$. The mitigating action associated to the high pulsed current extraction is partially compensated but the higher electrolyte temperatures, and the associated impurities production, as discussed in Section 3.2.

On the other hand, Fig. 10 illustrates the comparison of the $C_{\mathrm{SC}}$ evolution during a LE stress at $328.15 \mathrm{~K}$, a PC stress at 298,15 K and a combined PC and LE stress at $328.15 \mathrm{~K}$. In this figure and in the following ones, the $C_{\mathrm{SC}}$ and ESR have been expressed in per unit of the correspondent starting value.

By observing Fig. 10 it is possible to make some considerations: (i) during a PC the capacitance fading increases if the temperature increases; (ii) at high temperature the LE ageing is not involving the highest $C_{\mathrm{SC}}$ fading. In what follows the physical explanations of these two experimental findings are given in Fig. 9. During the first hours of cycling $(25 \mathrm{~h})$ the $C_{\mathrm{SC}}$ fading of the PC is practically independent on the temperature. It is possible to assume that the first porosity changes appearing on the electrode surfaces are independent from the value of the temperature. The new impurities produced by the temperature itself are not enough to contribute significantly to the porosity changes (in this respect for the same observed time window the $C_{\mathrm{SC}}$ fading during the LE stress is lower). After a certain time, in our case $25 \mathrm{~h}$, the effect of the high temperature along with the PC stress is showing its effect. The result, due to solvent losses and the increase of impurities creation, is the intensification of the ageing process and, consequently, the acceleration of the $C_{\mathrm{SC}}$ fading. After $100 \mathrm{~h}$ of PC at $328.15 \mathrm{~K}$ or at $298.15 \mathrm{~K}$ the difference in the $C_{\mathrm{SC}}$ fading is equal to $2 \%$. This

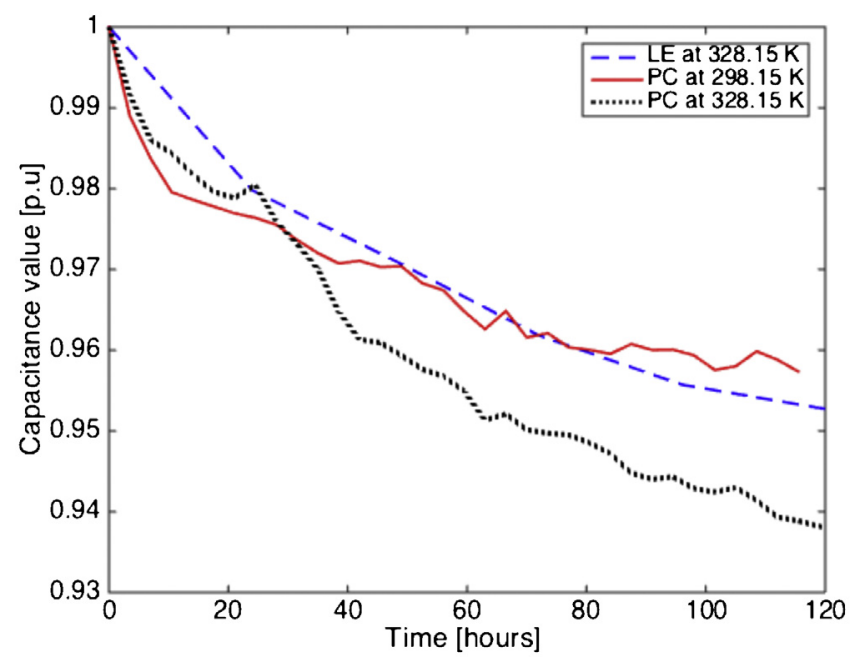

Fig. 11. $C_{\mathrm{SC}}$ evolution during combined PC-LE test. The results for each type of test report the average $C_{\mathrm{SC}}$ obtained on the fours SC samples under test.

difference is considerable since the majority of the SC manufactures declares the duration of the SC lifetime with the capacitance reduction of $20 \%$. Moreover, the $C_{\mathrm{SC}}$ reduction is less important during a LE stress since the ageing mechanism associated with the porosity change is not taking place. Additionally, the extraction of the current during a combined PC and LE stress is producing an over temperature involving a higher solvent evaporation and consequently an acceleration of the capacitance fading.

Fig. 11 illustrates the comparisons of ESR evolution versus the delivered charge during a LE stress at $328.15 \mathrm{~K}$, a combined PC and LE stress at $328.15 \mathrm{~K}$ and a PC stress at $298.15 \mathrm{~K}$. By observing Fig. 11, it is possible to make the following considerations: (i) during a PC the ESR increase is considerable if the temperature increases; (ii) at high temperature the LE ageing is not involving the highest ESR increase. In what follows the physical explanations of these two experimental findings. During the first $20 \mathrm{~h}$ the ESR p.u. value measured during the PC test and the ageing stress are practically the same. Then, as already explained, the extraction of the current with the associate over-temperature involves a decrease of the ESR value during the PC test at $298.15 \mathrm{~K}$. The LE stress is more critical since the temperature of $328.15 \mathrm{~K}$ involves impurities production and solvent evaporation. The combined PC and LE stress at $328.15 \mathrm{~K}$

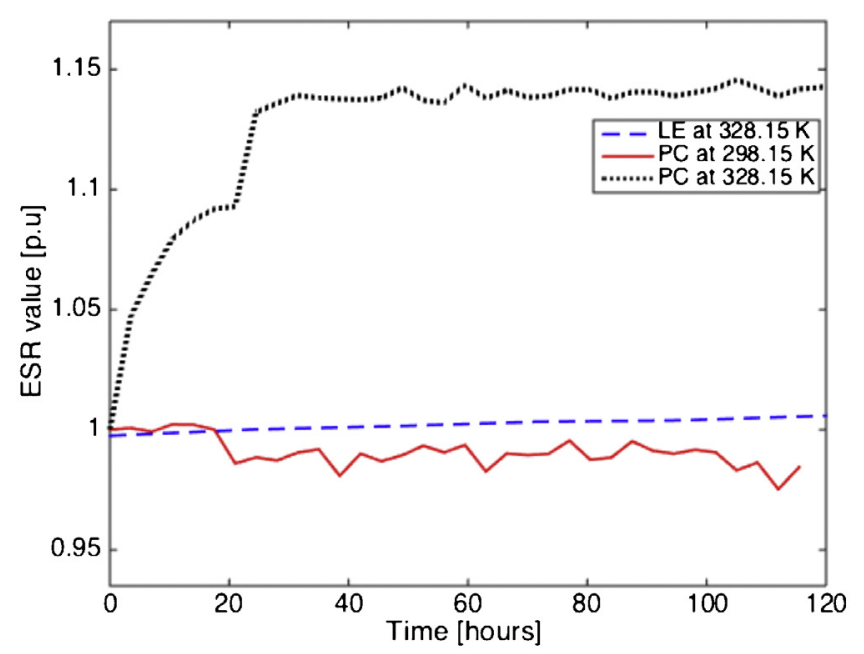

Fig. 12. ESR evolution during combined PC-LE test. The results for each type of test report the average ESR obtained on the fours SC samples under test. 
combined the disadvantages of both ageing processes: solvent evaporation and impurities production along with porosity changes. The associated ESR evolution is, consequently, the worst one and characterized by a sudden increase during the first $20 \mathrm{~h}$. This could be related to the sudden impurities production amplified by both current extraction and high temperature (Fig. 12).

\section{Conclusions}

The work here presented has described the ageing mechanisms involving the performances-fading of SCs. In particular, this study has been carried out by means of an experimental characterization and physical considerations.

The main originalities and experimental findings are:

- a comprehensive analysis of the state-of-art related to the description of ageing processes in electrochemical devices that allowed for creating a link between: (i) internal SC micro variables (porosity of the electrode surface, diffusion and conductivity of the electrolyte) and (ii) external SC macro variables (temperature, applied voltage and delivered current);

- from an experimental point of view, the paper has illustrated and discussed the performances fading (in terms of $C_{\mathrm{SC}}$ decrease and ESR increase) when the targeted SCs are cycled with high-pulsed values of current (above the device rated current) and hightemperature that represent typical operating conditions of these devices.

- The experimental investigation allowed to find that the ESR value does not increase considerably during a PC stress at nominal temperature. This is supported by the fact that the extraction of high-pulsed current allows for partially removing the impurities blocking charge extraction from the pores of the electrode surface.

The second part of the work will make use of the experimental findings and phenomenological observations summarized above to define a model capable to predict/quantify the State-of-Health of the SC.

\section{Acknowledgment}

The authors thankfully acknowledge the Swiss Innovation and Technology Committee (CTI) since the scientific activity described in this paper is framed within the context of the researches of the Swiss Competence Center for Energy Research "FURIES" (Future Swiss Electrical Infrastructure).

\section{Appendix A. State of art of accelerated SC ageing test}

\section{State of art of accelerated SC ageing test}

The aim of this Appendix is to show that the test had run enough time to reach the equivalent maximum number of cycles close to the manufacturer's specification. In order to reach this number of cycles, it is necessary to:

- account for the accelerated ageing effects of temperature and current (in our work we used temperature and current above the rated ones);

- account for the recovering effect associated to a voltage lower than the rated one (in our work we used a voltage of $2.45 \mathrm{~V}$ ).

Concerning the temperature effect during a LE stress, the available literature (e.g., [4,5]) agrees on the fact that an average increase of $7^{\circ} \mathrm{C}$ of the SC temperature reduces the expected lifetime of a factor 2 .
Concerning the voltage effect during a LE stress, the same type of life reduction can be achieved via an increased SC voltage of $120 \mathrm{mV}[4,5]$. It is worth observing that the opposite effect is obtained for a voltage reduction.

Concerning the effects pf the current extraction during a PC test, the main parameters affecting the lifetime are: (a) the RMS current value; (b) its peak value; (c) its waveshape.

The literature that studied the acceleration of the SC ageing introduced by delivered currents with RMS values much higher than the rated ones is quite scarce $[7,30,31]$. In these works, it is possible to observe the same results of the above rule of thumb concerning the performances fading during combined LE and PC stresses. The Authors of [29] detected an accelerated ageing factor equal to 1.8 for any increase of $10^{\circ} \mathrm{C}$ of temperature in the range $20^{\circ} \mathrm{C}-60^{\circ} \mathrm{C}$, during combined PC and LE stresses.

Ref. [7] illustrates the effect of different current peak values having the same RMS value. They compared all the proposed tests with the performance fading achieved with the nominal RMS value, $I_{\mathrm{RMSN}}$. The main findings can be summarized as follows. For any PC stress, if the peak current value has a magnitude of $2 \mathrm{I}_{\mathrm{RMSN}}$, the performances fading, in term of $C_{\mathrm{SC}}$ decrease, is augmented of a factor equal to 1.2 after 25,000 cycles. In this respect, we can keep the same relationship even for an higher number of cycles (in this first part of the manuscript we shown in Fig. 11 that for higher number of cycles the relative difference on $C_{\mathrm{SC}}$ decrease, at different average temperatures, becomes higher for higher number cycles).

The above literature summary on SC accelerated ageing, allows for computing an equivalent number of our cycles referred to the nominal operating conditions.

\section{Equivalent cycles computation}

In our case, we ran combined $\mathrm{LE}$ and $\mathrm{PC}$ tests at $55^{\circ} \mathrm{C}$ for 60 days with a charging current of $10 \mathrm{~A}$ during $7 \mathrm{~s}$ and a discharge current of $165 \mathrm{~A}$ for $1.5 \mathrm{~s}$. The associated RMS value is $37 \mathrm{~A}$, close to the rated one (36A). During this period, we observed an exponential decay of the $C_{\mathrm{SC}}$ value and, then, a quasi linear evolution of such a degradation. The number of discharging cycles performed during this time window was equal to 185,000 .

The equivalent number of cycles $N_{\mathrm{EQ}}$ referred to the nominal operating conditions should take into account the difference in average temperatures, average voltage and current peak values as summarized in the previous sub-section.

The manufacture declares half millions of cycle at $25^{\circ} \mathrm{C}, 2.7$ as rated voltage and RSM current value equal to 36A.

In our case, we have a different temperature and, based on Ref. [31] we should introduce the accelerating factor $K_{\mathrm{T}}$ accounting for overtemperature effects that can be computed as:

$K_{\mathrm{T}}=1.8^{\frac{T_{\mathrm{SCC}}}{\mathrm{T}_{\mathrm{SCN}}}}=1.8^{\frac{55^{\circ} \mathrm{C}}{25^{\circ} \mathrm{C}}}=5.83$

In our case, we have a lower voltage and, based on reference $[4,5]$ we should introduce the mitigating ageing factor $K_{\mathrm{V}}$ accounting for undervoltage effects (there is no other reference accounting for voltage effect during PC test):

$K_{\mathrm{V}}=2^{\frac{V_{\mathrm{N}}-V_{\mathrm{SC} i}}{120 \mathrm{mV}}}=2^{\frac{2.7-2.45}{120 \mathrm{mV}}}=4.23$

where $V_{\mathrm{SCi}}$ is the used voltage.

In our case, we have an RMS value practically equal to the nominal one but with a peak value, $I_{\text {PEAK, }}$ and a shape different from the rated one. Based on reference [7], we should introduce the accelerated ageing factor $K_{\mathrm{P}}$ accounting for peak-current values:

$K_{\mathrm{P}}=1.2^{\frac{I_{\mathrm{PEAK}}}{\text { RMSN }}}=1.2^{\frac{165}{36}}=2.306$ 
Unfortunately, even if the available literature declares and accelerated ageing factor due to the current shape (from a constant current profile to an irregular current profile) they do not give details on its computation. In this respect, we have disregarded this factor in this computation.

The equivalent number of cycles is the computed as:

$N_{\mathrm{EQ}}=N_{i} \times \frac{K_{\mathrm{T}}}{K_{\mathrm{V}}} \times K_{\mathrm{P}}=185000 \times \frac{5.83}{4.23} \times 2.306=586450$

where $N_{i}$ is the number of cycles performed at $V_{\mathrm{SCi}}, T_{\mathrm{SCi}}$ and with $I_{\text {PEAK. }}$

\section{References}

[1] Y. Aiping, V. Chabot, J. Zhang, Electrochemical Supercapacitors for Energy Storage and Delivery, CRC Press, 2012, pp. 3.

[2] C. Lin, B. Popov, H.J. Ploehnz, Modeling the effects of electrode composition and pore structure on the performance of electrochemical capacitors, J. Electrochem. Soc. 149 (2) (2002) 167-175.

[3] V. Srinivasan, J. Weidner, Mathematical modeling of electrochemical capacitors, J. Electrochem. Soc. 146 (5) (1999) 1650-1658.

[4] Oliver Bohlen, Julia Kowal, Dirk Uwe Sauer, Ageing behaviour of electrochemical double layer capacitors. Part I: experimental study and ageing model, J. Power Sources 172 (2007) 468-475.

[5] Oliver Bohlen, Julia Kowal, Dirk Uwe Sauer, Ageing behaviour of electrochemical double layer capacitors. Part II: lifetime simulation model for dynamic applications, J. Power Sources 173 (2007) 626-632.

[6] D. Linzen, S. Buller, E. Karden, R.W. De Doncker, Modeling the dynamic behavior of supercapacitors using impedance spectroscopy, IEEE Trans. Ind. Appl. 41 (5) (2005) 1135-1141 September-October.

[7] O. Briat, J.-M. Vinassa, N. Bertrand, H. El Brouji, J.-Y. Dele’tage, E. Woirgard, Contribution of calendar ageing modes in the performances degradation of supercapacitors during power cycling, Microelectron. Reliab. 50 (2010) 17961803.

[8] A. Hammar, P. Venet, R. Lallemand, G. Coquery, G. Rojat, Study of accelerated aging of supercapacitors for transport applications, IEEE Trans. Ind. Electron. 57 (December (12)) (2010) 3972-3979.

[9] R. Kötz, M. Hahna, R. Gallay, R. Kötz, M. Hahna, R. Gallay, Temperature behavior and impedance fundamentals of supercapacitors, J. Power Sources 154 (March (2)) (2006) 550-555.

[10] O. Briat, J.-M. Vinassa, N. Bertrand, H. El Brouji, E. Woirgard, Impact of calendar life and cycling ageing on supercapacitor performance, IEEE Trans. Veh. Technol. 58 (October (8)) (2009) 3917-3929.

[11] N. Rizoug P. Bartholomeus, P. Le Moigne, Study of the ageing process of a supercapacitor module using direct method of characterization, IEEE Trans. Energy Convers. 27 (June (2)) (2012) 220-228.

[12] O. Chaari, J.Y. Briat, Performances regeneration of supercapacitors during accelerated ageing tests in power cycling, Power Electron. Appl. (EPE) (2011) 1-7 August 30, 2011-September 1, 2011.

[13] W. Lajnef, J. Vinassa, O. Briat, H. El Brouji, S. Azzopardi, E. Woirgard, Quantification of ageing of ultracapacitors during cycling tests with current profile characteristics of hybrid and electric vehicles applications, IET Electr. Power Appl. 1 (September (5)) (2007) 683-689.
[14] P. Azaïsa, L. Duclauxa, P. Florianb, D. Massiotb, M. Rodenasc, A. Solanoc, J.P. Peresd, C. Jehouletd, F. Béguin, J. Power Sources 171 (2007) 1046-1053.

[15] P. Kurzweil, M. Chwistek, Calendar ageing and health diagnosis of supercapacitor, J. Power Sources 176 (2008) 555-567.

[16] A. Lewandowski, M. Galinski, Practical and theoretical limits for electrochemical double-layer capacitors, J. Power Sources 173 (2007) 822-828.

[17] Makoto Ue, Kazuhiko Ida, Shoichiro Mori, Electrochemical properties of organic liquid electrolytes based on quaternary onium salts for electrical double-layer capacitors, J. Electrochem. Soc. 141 (11) (1994) 2989-2996.

[18] Kedi Cai, Haijing Jiang, Wiehua Pu, Comparative investigation of organic solution and ionic liquid as electrolyte under lithium-air battery nt, J. Electrochem. Sci. 9 (2014) 390-397.

[19] D. Torregrossa, M. Paolone, Novel experimental investigation of supercapacitor ageing during combined life-endurance and power-cycling tests, IECON 2013, (2013) November.

[20] D. Torregrossa, K.E. Toghill, V. Amstutz, H.G. Hubert, M.P. irault, aolone, Macroscopic indicators of fault diagnosis and ageing in electrochemical double layer capacitors, J. Energy Storage 2 (August) (2015) 8-24.

[21] R. German, A. Saria, P. Veneta, O. Briat, J.-M. Vinassa, Study on specific effects of high frequency ripple currents and temperature on supercapacitors ageing, Microelectron. Reliab. 55 (August-September (9-10)) (2015) 2027-2031.

[22] T. Kovaltchouk, B. Multon, H. Ben Ahmed, J. Aubry, P. Venet, Enhanced aging model for supercapacitors taking into account power cycling: application to the sizing of an energy storage system in a direct wave energy converter, IEEE Trans. Ind. Appl. 51 (May-June (3)) (2015) 2405-2414.

[23] R. German, A. Hammar, R. Lallemand, A. Sari, P. Venet, Novel experimental identification method for a supercapacitor multipore model in order to monitor the state of health, IEEE Trans. Power Electron. 31 (January (1)) (2016) $548-559$.

[24] J. Vetter, P. Novák, M.R. Wagner, C. Veit, K.-C. Möller, J.O. Besenhard, Ageing mechanisms in lithium-ion batteries, J. Power Sources 147 (September (1-2)) (2005) 269-281.

[25] M. Marracci, B. Tellini, M. Catelani, L. Ciani, Ultracapacitor degradation state diagnosis via electrochemical impedance spectroscopy, IEEE Trans. Instrum. Meas. 64 (July (7)) (2015) 1916-1921.

[26] R. Germana, A. Saria, P. Veneta, M. Ayadib, Briat, J.M. Vinassa, Prediction of supercapacitors floating ageing with surface electrode interface based ageing law, Microelectron. Reliab. 54 (September-October (9-10)) (2014) 1813-1817.

[27] P. Azais, M. Lillo-Rodenas, A. Linares-Solano, J.P. Peres, L. Duclaux, F. Beguin, Causes of ageing of supercapacitors based on activated carbon electrodes and organic electrolyte, Improvement of the electrochemical characteristics by post-treatments of activated carbon.

[28] D. Cericola, R. Kötz, A. Wokaun, Effect of electrode mass ratio on aging of activated carbon based supercapacitors utilizing organic electrolytes, J. Power Sources 196 (March (6)) (2011) 3114-3118.

[29] P.W. Ruch, D. Cericola, A. Schimt, R. Kötz, A. Wokaun, A comparison of the aging of electrochemical double layer capacitors with acetonitrile and propylene carbonate-based electrolytes at elevated voltages electrodes degradations, Electrochim. Acta 55 (February (7)) (2010) 2352-2357.

[30] K. Paul, M. Christian, V. Pascal, C. Guy, R. Gerard, Z. Younes, Constant power cycling for accelerated ageing of supercapacitors, EPE '09. 13th European Conference on Power Electronics and Applications 1 (September (10)) (2015) $8-10$.

[31] M. Uno, K. Tanaka, Accelerated ageing testing and cycle life prediction of supercapacitors for alternative battery applications, Telecommunications Energy Conference (INTELEC), 011 IEEE 33rd International, 12011, pp. 9-13 October. 